\title{
Impact of Self and Co-Channel Interference on An DNF Full-Duplex One-Way Relay System
}

\author{
Bilal A. Jebur*†, Charalampos C. Tsimenidis* \\ ${ }^{*}$ School of Electrical and Electronic Engineering, Newcastle University \\ Newcastle upon Tyne, Merz Court, NE1 7RU, UK \\ $\dagger$ College of Electronic Engineering, Ninevah University, Iraq \\ Email: \{Bilal.Jebur, Charalampos.Tsimenidis\}@ newcastle.ac.uk
}

\begin{abstract}
In this paper, we study the impact of the co-channel interference $(\mathrm{CCI})$ in conjunction with the impact of residual self-interference (SI) on the end-to-end (E2E) performance of a denoise-and-forward full-duplex one-way relay channel (DNFFD-OWRC) network. The investigated system comprises one source node $S$, which communicates with one destination node $D$ with the aid of an FD relay node, over Rayleigh fading channels. Closed-form expressions for the cumulative distribution function (CDF) and the distribution of the E2E signal-to-interference and noise ratio (SINR) are derived and presented. Moreover, a closedform expression for the E2E outage probability is presented and validated using Monte Carlo simulations. The obtained results demonstrate the impact of the residual SI and CCI on the E2E performance of the DNF-FD-OWRC and demonstrate the ability of DNF-OWRC to improve the throughput of a conventional half-duplex (HD) OWRC.

Index Terms-co-channel interference (CCI), self-interference (SI), denoise-and-forward full-duplex one-way relay channel (DNF-FD-OWRC), end-to-end (E2E), cumulative distribution function (CDF), signal-to-interference and noise ratio (SINR), half-duplex (HD)
\end{abstract}

\section{INTRODUCTION}

The demand for wireless communication technologies that can meet the ever-increasing throughput requirements for the next generation communication systems has dramatically increased [1]. Hence, recently full-duplex (FD) cooperative systems have gained immense interest due to their ability to approximately double the throughput of the traditional half-duplex (HD) cooperative system. However, FD systems suffer from a tremendous challenge, i.e. self-interference (SI), which limits their performance gain. Consequently, various self-interference cancellation (SIC) schemes have been presented to tackle this challenge. A passive SIC scheme, which employs antenna separation and antenna directional isolation, was presented in [2]. Next, analog and digital SICs were introduced and investigated in [3] and [4], respectively. Moreover, a hybrid SIC system, which comprises adaptive radio frequency (RF) cancellation and digital cancellation schemes, was proposed in [5]. Next, an active RF cancellation algorithm that exploits adaptive nonlinear filtering to attain effective SI cancellation when a nonlinear power amplifier (PA) is used, was presented in [6].

\section{A. Related Work}

An extensive survey on FD relay channel issues and challenges for different relaying schemes was presented in
[7]. Moreover, an orthogonal frequency-division multiplexing (OFDM) based FD physical-layer network coding (PLNC) system was introduced in [8]. In [9], the ability to exploit the direct path in amplify-and-forward (AF) FD relaying systems to improve the end-to-end (E2E) performance was examined. Next, in [10], the outage probability of an AF-FDPLNC system was investigated. In [11], a tight upper bound ergodic capacity expression was presented and exploited to examine the end-to-end (E2E) ergodic capacity of an AF-FDPLNC system. Next, in [12], the performance of a MIMO-FDOWRC network was investigated in the presence of the CCI interference, however, the authors in this work considered the $\mathrm{CCI}$ at the relay only and the CCI presence at the destination node was not considered.

\section{B. Contribution}

In this paper, we investigate the performance of an FDOWRC network with one source node, one destination node and one denoise-and-forward (DNF) FD relay node in the presence of CCI at the relay and destination node in conjunction with the presence of the residual SI at the relay. Moreover, the performance of considered FD-OWRC is compared with that of the HD-OWRC to examine the throughput gain that can be attained by using FD relays. The main contributions of this work are as follows:

- We examine the E2E performance of the considered DNF-FD-OWRC system, in which the nodes are communicating over reciprocal and asymmetric Rayleigh fading channels.

- Closed-form expressions for the probability density function (PDF) and cumulative distribution function (CDF) of the E2E signal-to-interference and noise ratio (SINR) for the considered system are presented in this paper. These expressions are very useful to derive closed-form expressions for the outage probability, average error rate and ergodic capacity of the DNF-FD-OWRC in the presence of CCI and residual SI.

- An E2E outage probability expression for the examined DNF-FD-OWRC is derived and confirmed by Monte Carlo simulation results.

\section{Paper Organization}

The remainder of the paper is organized as follows: The system model is illustrated in Section II. Section III presents 
the derivation of the SINR and the statistical analysis of the system under investigation. The analytical and simulation based results of the investigated system are presented in Section IV. Finally, the conclusions of this paper are presented in Section V.

\section{SySTEM MODEL}

This section presents the DNF-FD-OWRC network with one source node, $S$, one destination node, $D$ and an FD relay node, $R$, as depicted in Fig. 1. We assume that there is no direct path connecting the source and the destination nodes, $S$ and $D$, subsequently, these nodes communicate with the assistance of the DNF-FD relay node. Moreover, we assume the presence of $N$ and $M$ co-channel interferences that affect the relay and destination node, respectively.

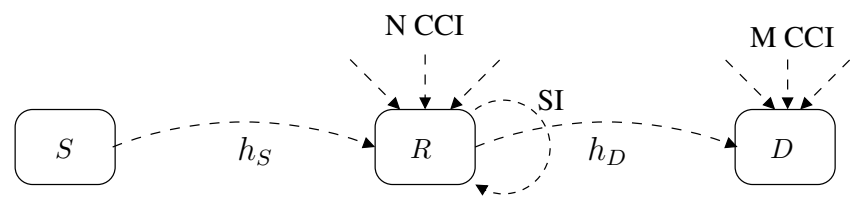

Fig. 1: Full-duplex one-way relay channel(FD-OWRC) system.

As the relay node in the examined FD-OWRC operates in FD mode, i.e. transmits and receives simultaneously, there will be self-interference (SI) swamping the signal received from the source node. However, the experimental results in [2]-[6], [13]-[15] have demonstrated that SI signal can be efficiently suppressed. Hence, the residual self-interference (RSI) should be included in the description of the received signal at the relay, which is given as

$$
y_{R}=x_{S} h_{S}+R S I_{R}+\sum_{n}^{N} x_{C R_{n}} h_{C R_{n}}+w_{R},
$$

where $x_{S}$ is the signal transmitted from the source node, $h_{S}$ represents the Rayleigh fading channel between the source and the relay and $R S I_{R}$ is the residual self-interference signal at the relay, which is modelled using a zero-mean complex-valued Gaussian PDF, i.e. $\mathcal{C N}\left(0, \sigma_{S I_{R}}^{2}\right)$, [12], [16][18]. Furthermore, $x_{C R_{n}}$ denotes the signal transmitted from the $n^{t h}$ interference source, $h_{C R_{n}}$ represents the Rayleigh fading channel between the $n^{t h}$ interference source and the relay. Moreover, $w_{R}$ denotes the additive white Gaussian noise (AWGN) samples at the relay node exhibiting a complex-valued circular Gaussian distribution, whose PDF is $\mathcal{C N}\left(0, N_{0}^{R}\right)$. Next, to obtain the signal to be transmitted from the relay to the end node, i.e. $x_{R}$, a DNF operation is performed on the received signal. The denoised signal at the relay, $x_{R}$ is obtained using a maximum-likelihood (ML) detection such that

$$
x_{R}=\underset{(q) \in \mathbb{Z}_{M}}{\operatorname{argmin}}\left|y_{R}-\left(h_{S} \mathcal{C}(q)\right)\right|^{2},
$$

where $\mathcal{C}$ represents the Quadrature Phase Shift Keying (QPSK) constellation vector. It is worth pointing out that compared to the time slot, in which a packet of symbols is transmitted, the denoising delay at the relay node is short enough. Hence, the effect of this denoising delay on the achievable rate is insignificant.

The received signal at the destination node is given as

$$
y_{D}=x_{R} h_{D}+\sum_{m}^{M} x_{C D_{m}} h_{C D_{m}}+w_{D},
$$

where $h_{D}$ and $h_{C D_{m}}$ are the Rayleigh fading channels between the relay and the destination node and between the $m^{t h}$ interference source and destination node, respectively. Moreover, $x_{C D_{m}}$ is the interference signal from the $m^{t h}$ interference source and $w_{D}$ represents the complex-valued AWGN with $\mathcal{C N}\left(0, N_{0}^{D}\right)$.

\section{STATISTICAL ANALYSIS AND PERFORMANCE EVALUATION}

In this section, we evaluate the PDF and CDF of the SINR at the relay and destination nodes along with the PDF and $\mathrm{CDF}$ of the E2E SINR. Moreover, the exact E2E outage probability of the DNF-FD-OWRC is derived in this section. The SINR at the relay is given as

$$
\gamma_{R}=\frac{\Lambda_{R}}{\epsilon_{R}+\sum_{n}^{N} \varepsilon_{R_{n}}+1}
$$

where $\Lambda_{R}=\frac{E_{S}\left|h_{S}\right|^{2}}{N_{0}^{R}}, \epsilon_{R}=\frac{\left|R S I_{R}\right|^{2}}{N_{0}^{R}}$ and $\varepsilon_{R_{n}}=\frac{E_{C R_{n}}\left|h_{C R_{n}}\right|^{2}}{N_{0}^{R}}$ denote the signal-to-noise ratio (SNR) at the relay, selfinterference-to-noise ratio and co-channel interference-tonoise ratio from the $n^{t h}$ interference source, respectively. Furthermore $E_{S}$ and $E_{C R_{n}}$ denote the average transmission energy at the source node and the $n^{t h}$ interference source, respectively. Next, the SINR at the destination node is given as

$$
\gamma_{D}=\frac{\Lambda_{D}}{\sum_{m}^{M} \varepsilon_{D_{m}}+1},
$$

where $\Lambda_{D}=\frac{E_{R}\left|h_{D_{l}}\right|^{2}}{N_{0}^{D}}$ and $\varepsilon_{D_{m}}=\frac{E_{C D_{m}}\left|h_{C D_{m}}\right|^{2}}{N_{0}^{D}}$ are the SNR at the destination node and co-channel interference-to-noise ratio from the $m^{t h}$ interference source, respectively. Moreover, $E_{R}$ and $E_{C D_{m}}$ are the average transmission energy at the relay and the $m^{t h}$ interference source, respectively. A closer investigation of (4) and (5) reveals that $\gamma_{R}$ and $\gamma_{D}$ can be presented as

$$
\gamma_{R}=\frac{X_{R}}{Y_{R}+Z_{R}+1}
$$

and

$$
\gamma_{D}=\frac{X_{D}}{Z_{D}+1}
$$

where $X_{R}=\Lambda_{R}, X_{D}=\Lambda_{D}, Y_{R}=\epsilon_{R}, Z_{R}=\sum_{n}^{N} \varepsilon_{R_{n}}$ and $Z_{D}=\sum_{m}^{M} \varepsilon_{D_{m}}$.

Due to the fact that all the channels in the investigated system are assumed to be quasi-static Rayleigh fading channels and the RSI exhibits a complex-valued circular Gaussian distribution with $\mathcal{C N}\left(0, \sigma_{S I_{R_{l}}}^{2}\right)$, [16], [17] and [18], the PDFs of $X_{R}, X_{D}, Y_{R}, Z_{R}$ and $Z_{D}$ are given respectively as

$$
p_{X_{L}}\left(X_{L}\right)=\frac{e^{-\frac{X_{L}}{\varrho_{L}}}}{\varrho_{L}}
$$




$$
\begin{gathered}
p_{Y_{R}}\left(Y_{R}\right)=\frac{e^{-\frac{Y_{R}}{\alpha_{S I}}}}{\alpha_{S I}}, \\
p_{Z_{R}}\left(Z_{R}\right)=\frac{Z_{R}^{N-1}}{\Gamma(N)\left(\alpha_{C I_{R}}\right)^{N}} e^{-\frac{Z_{R}}{\alpha_{C I}}},
\end{gathered}
$$

and

$$
p_{Z_{D}}\left(Z_{D}\right)=\frac{Z_{D}^{M-1}}{\Gamma(M)\left(\alpha_{C I_{D}}\right)^{M}} e^{-\frac{Z_{D}}{\alpha_{C I}}},
$$

where $L \in\{R, D\}, \varrho_{R}=E\left(\Lambda_{R}\right)$ and $\varrho_{D}=E\left(\Lambda_{D}\right)$ denote the average SNR at the relay and destination nodes, respectively, and $\alpha_{S I}=E\left(\epsilon_{R}\right)$ is the average residual SI to noise ratio at the relay. Furthermore, $\alpha_{C I_{R}}=E\left(\varepsilon_{R_{n}}\right)$ and $\alpha_{C I_{D}}=E\left(\varepsilon_{D_{m}}\right)$ represent the average CCI to noise ratio at the relay and destination node, respectively.

Let $I_{t}$ represent the total interference-to-noise ratio (INR) at the relay, which results from $Y_{R}+Z_{R}$. Then, the PDF of the total INR can be evaluated using the integral in [19, Eq. (6.52)] such that

$$
p_{I_{t}}\left(I_{t}\right)=\int_{0}^{I_{t}} p_{Z_{R}}\left(Z_{R}\right) p_{Y_{R}}\left(I_{t}-Z_{R}\right) d Z_{R} .
$$

Using (9) and (10) to substitute $p_{Y_{R}}\left(Y_{R}\right)$ and $p_{Z_{R}}\left(Z_{R}\right)$ in (12), respectively, results in

$$
p_{I_{t}}\left(I_{t}\right)=\frac{e^{-\frac{I_{t}}{\alpha_{S I}}}}{\Gamma(N) \alpha_{C I_{R}}^{N} \alpha_{S I}} \int_{0}^{I_{t}} Z_{R_{l}}^{N-1} e^{-\frac{\alpha_{S I}-\alpha_{C I}}{\alpha_{S I} \alpha_{C I}} Z_{R}} d Z_{R} .
$$

This integral can be solved by exploiting the result in [20, Eq. (3.351.1)], which yields

$$
p_{I_{t}}\left(I_{t}\right)=\frac{e^{-\frac{I_{t}}{\alpha_{S I}}} \alpha_{S I}^{N-1}}{\Gamma(N)\left(\alpha_{S I}-\alpha_{C I_{R}}\right)^{N}} \gamma\left(N, \frac{\alpha_{S I}-\alpha_{C I_{R}}}{\alpha_{S I} \alpha_{C I_{R}}} I_{t}\right),
$$

where $\gamma(.,$.$) denotes the lower incomplete Gamma function.$

Since $I_{t}=Y_{R}+Z_{R}$, (6) can be rewritten as

$$
\gamma_{R}=\frac{X_{R}}{I_{t}+1} .
$$

Hence the PDF of the SINR at the relay can be computed using the formula in [21, Eq. (4.6)] such that

$$
p_{\gamma_{R}}\left(\gamma_{R}\right)=\int_{0}^{\infty}\left(1+I_{t}\right) p_{X_{R}}\left(\left(1+I_{t}\right) \gamma_{R}\right) p_{I_{t}}\left(I_{t}\right) d I_{t} .
$$

Substituting (8) and (14) into (16) gives

$$
\begin{aligned}
p_{\gamma_{R}}\left(\gamma_{R}\right) & =\frac{\alpha_{S I}^{N-1} e^{-\frac{\gamma}{\varrho_{R}}}}{\Gamma(N)\left(\alpha_{S I}-\alpha_{C I_{R}}\right)^{N} \varrho_{R}}\left(\int_{0}^{\infty} e^{-\nu I_{t}} \gamma\left(N, \mu I_{t}\right) d I_{t}\right. \\
& \left.+\int_{0}^{\infty} I_{t} e^{-\nu I_{t}} \gamma\left(N, \mu I_{t}\right) d I_{t}\right)
\end{aligned}
$$

where $\nu=\frac{\left(\varrho_{R}+\gamma \alpha_{S I}\right)}{\varrho_{R} \alpha_{S I}}$ and $\mu=\frac{\alpha_{S I}-\alpha_{C I_{R}}}{\alpha_{S I} \alpha_{C I_{R}}}$. Moreover, the expressions in (17) can be re-arranged as

$$
\begin{aligned}
p_{\gamma_{R}}\left(\gamma_{R}\right) & =\frac{\alpha_{S I}^{N-1} e^{-\frac{\gamma}{\varrho_{R}}}}{\Gamma(N)\left(\alpha_{S I}-\alpha_{C I_{R}}\right)^{N} \varrho_{R}}\left(\frac{1}{\mu} \int_{0}^{\infty} e^{-\frac{\nu}{\mu} I_{t}} \gamma\left(N, I_{t}\right) d I_{t}\right. \\
& \left.+\frac{1}{\mu^{2}} \int_{0}^{\infty} I_{t} e^{-\frac{\nu}{\mu} I_{t}} \gamma\left(N, I_{t}\right) d I_{t}\right)
\end{aligned}
$$

so that the resulting first integral can be solved using [20, Eq. (6.451)] and the second integral can be solved by exploiting integration by parts, i.e. $\int u d v=u v-\int v d u$. Consequently, $p_{\gamma_{R_{l}}}(\gamma)$ can be expressed as

$$
\begin{aligned}
p_{\gamma_{R}}\left(\gamma_{R}\right) & =\frac{\varrho_{R}^{N} e^{-\frac{\gamma_{R}}{\varrho_{R}}}}{\left(\varrho_{R_{l}}+\gamma_{R} \alpha_{S I}\right)\left(\varrho_{R}+\gamma_{R} \alpha_{C I_{R}}\right)^{N}} \\
& +\frac{\varrho_{R}^{N+1} \alpha_{C I_{R}} e^{-\frac{\gamma_{R}}{\varrho_{R}}}}{\left(\varrho_{R}+\gamma_{R} \alpha_{S I}\right)\left(\varrho_{R}+\gamma_{R} \alpha_{C I_{R}}\right)^{N+1}} \\
& +\frac{\varrho_{R}^{N+1} \alpha_{S I} e^{-\frac{\gamma_{R}}{\varrho_{R}}}}{\left(\varrho_{R}+\gamma_{R} \alpha_{S I}\right)^{2}\left(\varrho_{R}+\gamma_{R} \alpha_{C I_{R}}\right)^{N}} .
\end{aligned}
$$

The CDF of the SINR at the relay can be obtained by using the integral in [21, Eq. (4.5)] such that

$$
P_{\gamma_{R}}\left(\gamma_{R}\right)=\int_{0}^{\infty} P_{X_{R}}\left(\left(1+I_{t_{l}}\right) \gamma_{R}\right) p_{I_{t}}\left(I_{t}\right) d I_{t}
$$

where $P_{X_{R}}\left(X_{R}\right)$ is the $\mathrm{CDF}$ of the $\mathrm{RV} X_{R}$, which is given as

$$
P_{X_{R}}\left(X_{R}\right)=1-e^{-\frac{X_{R}}{e_{R}}} .
$$

Using (21) and (14) to substitute $P_{X_{R}}\left(X_{R}\right)$ and $p_{I_{t}}\left(I_{t}\right)$ in (20), respectively, yields

$$
\begin{aligned}
P_{\gamma_{R}}\left(\gamma_{R}\right)= & \frac{\alpha_{S I}^{N-1}}{\Gamma(N)\left(\alpha_{S I}-\alpha_{C I_{R}}\right)^{N}}\left(\int_{0}^{\infty} e^{-\frac{I_{t_{l}}}{\alpha_{S I}}} \gamma\left(N, \mu I_{t}\right) d I_{t}\right. \\
& \left.-e^{-\frac{\gamma_{R}}{\varrho_{R}}} \int_{0}^{\infty} e^{-\nu I_{t}} \gamma\left(N, \mu I_{t}\right) d I_{t}\right)
\end{aligned}
$$

This integral can be re-arranged as

$$
\begin{aligned}
P_{\gamma_{R}}\left(\gamma_{R}\right)= & \frac{\alpha_{S I}^{N-1}}{\Gamma(N)\left(\alpha_{S I}-\alpha_{C I_{R}}\right)^{N}}\left(\frac{1}{\mu} \int_{0}^{\infty} e^{-\frac{I_{t}}{\mu \alpha S I}} \gamma\left(N, I_{t}\right) d I_{t}\right. \\
& \left.-\frac{e^{-\frac{\gamma_{R}}{\rho_{R}}}}{\mu} \int_{0}^{\infty} e^{-\frac{\nu}{\mu} I_{t}} \gamma\left(N, I_{t}\right) d I_{t}\right)
\end{aligned}
$$

in order to solve the resulting integrals using [20, Eq. (6.451)], which gives

$$
P_{\gamma_{R}}\left(\gamma_{R}\right)=1-\frac{\varrho_{R}^{N+1} e^{-\frac{\gamma_{R}}{\varrho_{R}}}}{\left(\varrho_{R}+\gamma_{R} \alpha_{S I}\right)\left(\varrho_{R}+\gamma_{R} \alpha_{C I_{R}}\right)^{N}} .
$$

On the other hand, since SINR at the destination node in (7) is given as a ratio of two random variables, the PDF of the SINR at the destination node can be evaluated using [21, Eq. (4.6)] such that

$p_{\gamma_{D}}\left(\gamma_{D}\right)=\int_{0}^{\infty}\left(1+Z_{D}\right) p_{X_{D}}\left(\left(1+Z_{D}\right) \gamma_{D}\right) p_{Z_{D}}\left(Z_{D}\right) d Z_{D}$

Next, substituting (8) and (11) in (25) yields



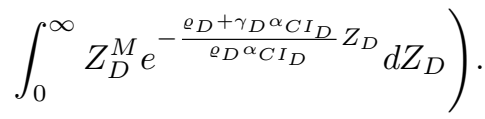


This integral can be solved by exploiting the result in [20, Eq. (3.326.2)], which results in

$$
p_{\gamma_{D}}\left(\gamma_{D}\right)=\frac{e^{\frac{-\gamma_{D}}{\varrho_{D}}} \varrho_{D}^{M-1}}{\left(\varrho_{D}+\gamma_{D} \alpha_{C I_{D}}\right)^{M}}+\frac{e^{\frac{-\gamma_{D}}{\varrho_{D}}} \varrho_{D}^{M} \alpha_{C I_{D}}}{\left(\varrho_{D}+\gamma_{D} \alpha_{C I_{D}}\right)^{M+1}}
$$

The CDF of the SINR at the destination node can be obtained by using [21, Eq. (4.5)] such that

$$
P_{\gamma_{D}}\left(\gamma_{D}\right)=\int_{0}^{\infty} P_{X_{D}}\left(\left(1+Z_{D}\right) \gamma_{D}\right) p_{Z_{D}}\left(Z_{D}\right) d Z_{D}
$$

where $P_{X_{D}}\left(X_{D}\right)$ is the $\mathrm{CDF}$ of the RV $X_{D}$, which is given as

$$
P_{X_{D}}\left(X_{D}\right)=1-e^{-\frac{X_{D}}{\varrho_{D}}} .
$$

Using (29) and (11) to substitute $P_{X_{D}}$ and $p_{Z_{D}}\left(Z_{D}\right)$ in (28), respectively, gives

$$
\begin{aligned}
P_{\gamma_{D}}\left(\gamma_{D}\right)= & \int_{0}^{\infty} \frac{Z_{D}^{M-1} e^{-\frac{Z_{D}}{\alpha_{C I}}}}{\Gamma(M) \alpha_{C I_{D}}^{M}} d Z_{D} \\
& -\frac{e^{\frac{-\gamma_{D}}{\varrho_{D}}}}{\Gamma(M) \alpha_{C I_{D}}^{M}} \int_{0}^{\infty} Z_{D}^{M-1} e^{-\frac{\varrho_{D}+\gamma_{D} \alpha_{C I}}{\varrho_{D} \alpha_{C I}} Z_{D}} .
\end{aligned}
$$

Exploiting [20, Eq. (3.326.2)] to solve the integrals in (30) yields

$$
P_{\gamma_{D}}\left(\gamma_{D}\right)=1-\frac{\varrho_{D}^{M} e^{\frac{-\gamma_{D}}{\varrho_{D}}}}{\left(\varrho_{D}+\gamma_{D} \alpha_{C I_{D}}\right)^{M}} .
$$

Finally, owing to the fact that the E2E SINR in the regenerative multihop relaying networks is dominated by the SINR of the weaker hop [22], the E2E SINR for the proposed system can be expressed as

$$
\gamma_{E 2 E}=\min \left(\gamma_{R}, \gamma_{D}\right)
$$

Hence, the PDF of the E2E SINR is given as

$$
\begin{aligned}
p_{\gamma_{E 2 E_{u p}}}(\gamma)= & p_{\gamma_{R}}(\gamma)+p_{\gamma_{D}}(\gamma)-\left[p_{\gamma_{R}}(\gamma) P_{\gamma_{D}}(\gamma)\right. \\
& \left.+p_{\gamma_{D}}(\gamma) P_{\gamma_{R}}(\gamma)\right] .
\end{aligned}
$$

Then, the PDF of the E2E SINR can be obtained by substituting (19), (24), (27) and (31) in (33), which yields

$$
\begin{aligned}
p_{\gamma_{E 2 E}}(\gamma) & =\left[\frac{e^{\frac{-\gamma_{D}}{\varrho_{D}}} \varrho_{D}^{M-1}}{\left(\varrho_{D}+\gamma_{D} \alpha_{C I_{D}}\right)^{M}}+\frac{e^{\frac{-\gamma_{D}}{\varrho_{D}}} \varrho_{D}^{M} \alpha_{C I_{D}}}{\left(\varrho_{D}+\gamma_{D} \alpha_{C I_{D}}\right)^{M+1}}\right] \\
& \times\left(\frac{\varrho_{R}^{N+1} e^{-\frac{\gamma}{\varrho_{R}}}}{\left(\varrho_{R}+\gamma \alpha_{S I}\right)\left(\varrho_{R}+\gamma \alpha_{C I}\right)^{N}}\right) \\
& +\left[\frac{\varrho_{R}^{N} e^{-\frac{\gamma}{\varrho_{R}}}}{\left(\varrho_{R_{l}}+\gamma \alpha_{S I}\right)\left(\varrho_{R}+\gamma \alpha_{C I_{R}}\right)^{N}}\right. \\
& +\frac{\varrho_{R}^{N+1} \alpha_{C I_{R}} e^{-\frac{\gamma}{\varrho_{R}}}}{\left(\varrho_{R}+\gamma \alpha_{S I}\right)\left(\varrho_{R}+\gamma \alpha_{C I_{R}}\right)^{N+1}} \\
& \left.+\frac{\varrho_{R}^{N+1} \alpha_{S I} e^{-\frac{\gamma}{\varrho_{R}}}}{\left(\varrho_{R}+\gamma \alpha_{S I}\right)^{2}\left(\varrho_{R}+\gamma \alpha_{C I_{R}}\right)^{N}}\right] \\
& \times\left(\frac{\varrho_{D}^{M} e^{\frac{-\gamma}{\varrho_{D}}}}{\left(\varrho_{D}+\gamma \alpha_{C I_{D}}\right)^{M}}\right)
\end{aligned}
$$

Furthermore, the CDF of the E2E SINR can be given as

$$
P_{\gamma_{E 2 E}}(\gamma)=P_{\gamma_{R}}\left(\gamma_{R}\right)+P_{\gamma_{D}}\left(\gamma_{D}\right)-P_{\gamma_{R}}\left(\gamma_{R}\right) P_{\gamma_{R}}\left(\gamma_{D}\right)
$$

Substituting (24) and (31) in (35), yields the CDF of the E2E SINR such that

$$
\begin{aligned}
P_{\gamma_{E 2 E}}(\gamma)=1- & \frac{\varrho_{R_{l}}^{N+1} \varrho_{D}^{M}}{\left(\varrho_{R_{l}}+\gamma \alpha_{S I_{l}}\right)\left(\varrho_{R_{l}}+\gamma \alpha_{C I_{R_{l}}}\right)^{N}} \\
& \times \frac{e^{-\frac{\left(\varrho_{R_{l}}+\varrho_{D}\right)}{\varrho_{R_{l}} \varrho_{D}}} \gamma}{\left(\varrho_{D}+\gamma \alpha_{C I_{D}}\right)^{M}} .
\end{aligned}
$$

The outage probability is defined as the probability of the SINR falling below a specific threshold, $\gamma_{t h}$ [23]. Hence, the E2E outage probability is given as

$$
\begin{aligned}
P_{\text {out }}^{E 2 E}\left(\gamma_{E 2 E_{t h}}\right) & \triangleq \operatorname{Pr}\left\{\gamma_{E 2 E} \leq \gamma_{E 2 E_{t h}}\right\} \\
& =\int_{0}^{\gamma_{E 2 E_{t h}}} p_{\gamma_{E 2 E}}\left(\gamma_{E 2 E_{t h}}\right) d \gamma
\end{aligned}
$$

The E2E outage probability is also expressed as

$$
P_{\text {out }}^{E 2 E}\left(\gamma_{E 2 E_{t h}}\right)=P_{\gamma_{E 2 E}}\left(\gamma_{E 2 E_{t h}}\right) \text {. }
$$

Since the outage probability definition is similar to that of the SINR CDF, the E2E outage probability can be obtained by evaluating (36) at $\gamma_{E 2 E_{t h}}$, which yields

$$
\begin{aligned}
P_{\gamma_{E 2 E}}\left(\gamma_{E 2 E_{t h}}\right)=1- & \frac{\varrho_{R_{l}}^{N+1} \varrho_{D}^{M}}{\left(\varrho_{R_{l}}+\gamma_{E 2 E_{t h}} \alpha_{S I_{l}}\right)\left(\varrho_{R_{l}}+\gamma_{E 2 E_{t h}} \alpha_{C I_{R_{l}}}\right)^{N}} \\
& \times \frac{e^{-\frac{\left(\varrho_{R_{l}}+\varrho_{D}\right)}{\varrho_{R_{l}} \varrho_{D}} \gamma_{E 2 E}{ }_{t h}}}{\left(\varrho_{D}+\gamma_{E 2 E_{t h}} \alpha_{C I_{D}}\right)^{M}} .
\end{aligned}
$$

\section{NumericAl Results}

In this section, we present the analytical and simulationbased results for the examined DNF-FD-OWRC system. We consider an uncoded FD-OWRC network with QPSK modulation, where two nodes communicate with the aid of a DNFFD relay node. First, the analytical E2E SINR distribution was derived and evaluated. Fig. 2, illustrate the impact of the residual SI on the distribution of the E2E SINR, whilst the CCI was fixed, $\varepsilon_{R}=0.001 \Lambda_{R}$, and the SNR was also fixed, $\Lambda_{R}=\Lambda_{D}=15 \mathrm{~dB}$. It can be observed from Fig. 2 that as the level of the residual SI increases the PDF of the SINR converges towards the low SINR region. Second, in order to have a better understanding of the impact of the residual SI and CCI on the performance of the investigated system, the analytical E2E outage probability was evaluated and validated by Monte Carlo simulations as shown in Figs. 3, 4 and 5.

Fig. 3 illustrates the analytical and simulation based outage probability results for the investigated system, i.e. DNF-FDOWRC, and those of the DNF-HD-OWRC, vs. SNR. These results were obtained with $N=M=1, \varepsilon_{R}=0.001 \Lambda_{R}$, $\varepsilon_{D}=0.001 \Lambda_{D}$, and three different scenarios of residual SI to noise ratio, $\epsilon_{R}=0,5,10 \mathrm{~dB}$, respectively, whilst the E2E SINR threshold, $\gamma_{E 2 E_{t h}}$, was fixed at $5 \mathrm{~dB}$. The comparison between the E2E outage probability results of the FD-OWRC and HD-OWRC reveals the impact of the residual SI on the performance of the system under investigation. In particular, it can be observed from Fig. 3 that there is an SNR penalty of 




Fig. 2: The PDF of the SINR at the destination node for DNFFD-OWRC with residual SI and different levels of IRI at SNR $=15 \mathrm{~dB}$.



Fig. 3: The analytical and simulation average E2E outage probability for DNF-HD-OWRC and DNF-FD-OWRC at $\gamma_{E 2 E_{t h}}=5 \mathrm{~dB}$.

$1.8,4.1$ and $7.8 \mathrm{~dB}$, respectively, when the residual SI to noise ratio level ranges from 0 to $10 \mathrm{~dB}$. This means that employing FD relays to double the throughput of the OWRC networks comes with an SNR cost, which varies with the residual SI to noise ratio levels. Moreover, the results in Fig. 3 show an error floor of $6.5 \times 10^{-3}$ due to the co-channel interference.

Figs. 4 and 5 show the impact of CCI on the E2E performance of the DNF-FD-OWRC network. Fig. 4 presents the



Fig. 4: The analytical and simulation average E2E outage probability for the DNF-FD-OWRC at $\gamma_{E 2 E_{t h}}=5 \mathrm{~dB}$.

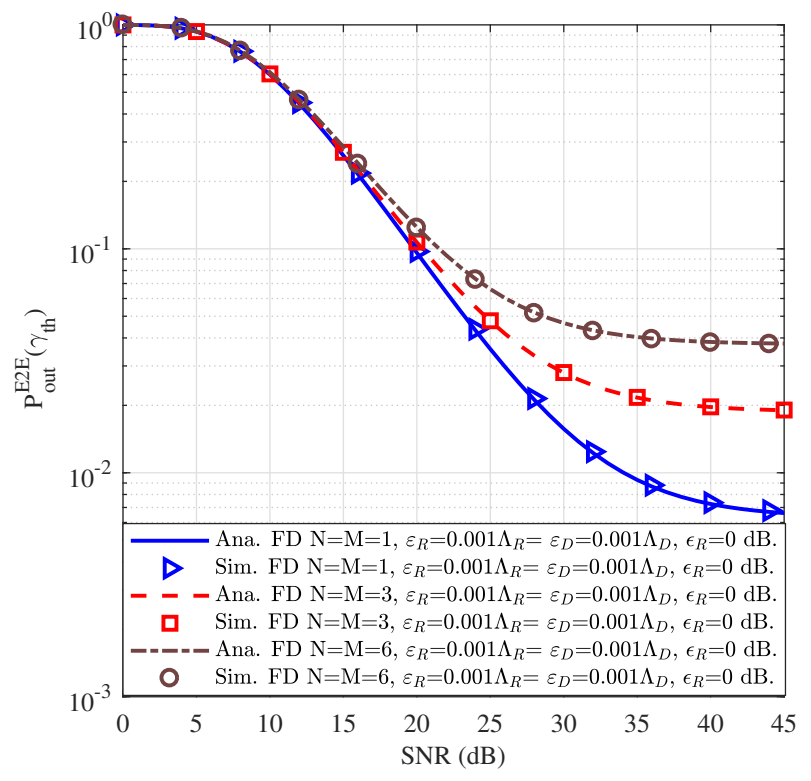

Fig. 5: The analytical and simulation average E2E outage probability for the DNF-FD-OWRC at $\gamma_{E 2 E_{t h}}=5 \mathrm{~dB}$.

E2E outage probability results of the DNF-FD-OWRC system with different CCI powers, whilst the residual SI to noise ratio was fixed, $\epsilon_{R}=0 \mathrm{~dB}$, and $N=M=1$. Moreover, it can be observed from Fig. 4 that the E2E outage probability of the system under investigation exhibits error floors of $6.5 \times 10^{-3}$, $3 \times 10^{-2}$ and $6 \times 10^{-2}$ for $\varepsilon_{R}=(0.001,0.005,0.01) \Lambda_{R}$ and $\varepsilon_{D}=(0.001,0.005,0.01) \Lambda_{D}$, respectively. Fig. 5 shows the E2E outage probability results of the DNF-FD-OWRC for different number of CCI, i.e. $N=M=1,3$ and 6 , while the residual SI to noise ratio was fixed, $\epsilon_{R}=0 \mathrm{~dB}$, and CCI 
power was also fixed, $\varepsilon_{R}=0.001 \Lambda_{R}$ and $\varepsilon_{D}=0.001 \Lambda_{D}$. The E2E results in Fig. 5 show error floors of $6.5 \times 10^{-3}$, $2 \times 10^{-2}$ and $4 \times 10^{-2}$ for $N=M=1,3$ and 6 , respectively. Furthermore, a closer examination of Figs. 4 and 5 reveals that the CCI has more impact on the E2E performance of the DNF-FD-OWRC systems than the residual SI. This comes from the fact that the residual SI affects the relay only, while CCI affects the relay and the destination node.

\section{Conclusions}

In this paper, we examined the E2E performance of an FD-OWRC, which consists of one source node transmitting information to one destination node with the assistance of an FD relay node over reciprocal and asymmetric Rayleigh fading channels. In essence, the performance of the considered FD-OWRC system was investigated in the presence of residual SI at the relay and CCI at both of the relay and the destination node. First, closed-form expressions for the CDF and PDF of the SINR at the relay and destination node were derived and presented and then used to obtain the CDF and PDF expressions for the E2E SINR of the FD-OWRC system. Second, the E2E outage probability expression of the investigated system was presented and confirmed using Monte Carlo simulation results. The obtained results illustrated the impact of the residual SI and CCI on the performance of the studied system. Specifically, the obtained results showed that there is an SNR penalty which is a function of the residual SI to noise ratio and CCI to noise ratio levels. Hence, OWRC system designers have to consider these SNR penalties to meet the aim of the FD-OWRC networks, i.e. double the throughput of the traditional HD-OWRC networks.

\section{ACKNOWLEDGMENT}

This work was supported by EPSRC under project EP/R002665/1, Full-Duplex For Underwater Acoustic Communications. The authors would like to thank the Research Council for this funding.

\section{REFERENCES}

[1] B. A. Jebur, Full Duplex-transceivers: Architectures and Performance Analysis. Newcastle University, 2017.

[2] E. Everett, A. Sahai, and A. Sabharwal, "Passive self-interference suppression for full-duplex infrastructure nodes," IEEE Trans. Wireless Commun., vol. 13, no. 2, pp. 680-694, February 2014.

[3] M. Duarte, C. Dick, and A. Sabharwal, "Experiment-driven characterization of full-duplex wireless systems," IEEE Trans. Wireless Commun., vol. 11, no. 12, pp. 4296-4307, December 2012.

[4] E. Ahmed and A. Eltawil, "All-digital self-interference cancellation technique for full-duplex systems," IEEE Trans. Wireless Commun., vol. 14, no. 7, pp. 3519-3532, July 2015.

[5] D. Korpi, J. Tamminen, M. Turunen, T. Huusari, Y. S. Choi, L. Anttila, S. Talwar, and M. Valkama, "Full-duplex mobile device: pushing the limits," IEEE Commun. Mag., vol. 54, no. 9, pp. 80-87, September 2016.

[6] A. Kiayani, M. Z. Waheed, L. Anttila, M. Abdelaziz, D. Korpi, V. Syrjl, M. Kosunen, K. Stadius, J. Ryynnen, and M. Valkama, "Adaptive nonlinear RF cancellation for improved isolation in simultaneous transmitreceive systems," IEEE Trans. Microw. Theory Tech., vol. PP, no. 99, pp. 1-14, 2018.

[7] G. Liu, F. R. Yu, H. Ji, V. C. M. Leung, and X. Li, "In-band full-duplex relaying: A survey, research issues and challenges," IEEE Commun. Surveys Tuts, vol. 17, no. 2, pp. 500-524, Secondquarter 2015.
[8] B. A. Jebur and C. C. Tsimenidis, "Performance analysis of OFDMBased denoise-and-forward full-duplex PLNC with imperfect CSI," in IEEE International Conference on Communication Workshop (ICCW), June 2015, pp. 997-1002.

[9] D. P. M. Osorio, E. E. B. Olivo, H. Alves, J. C. S. S. Filho, and M. Latva-aho, "Exploiting the direct link in full-duplex amplify-andforward relaying networks," IEEE Signal Process. Lett., vol. 22, no. 10, pp. 1766-1770, Oct 2015.

[10] B. A. Jebur, C. C. Tsimenidis, M. Johnston, and J. Chambers, "Outage probability of an AF full-duplex physical-layer network coding system," in 2016 24th European Signal Processing Conference (EUSIPCO), Aug 2016, pp. $1828-1832$.

[11] B. A. Jebur, C. C. Tsimenidis, and J. A. Chambers, "Tight upper bound ergodic capacity of an AF full-duplex physical-layer network coding system," in 2016 IEEE 27th Annual International Symposium on Personal, Indoor, and Mobile Radio Communications (PIMRC), Sept 2016, pp. 1-6.

[12] A. Almradi and K. A. Hamdi, "MIMO full-duplex relaying in the presence of co-channel interference," IEEE Trans. Veh. Technol., vol. 66, no. 6, pp. 4874-4885, June 2017.

[13] J. I. Choi, M. Jain, K. Srinivasan, P. Levis, and S. Katti, "Achieving single channel, full duplex wireless communication," in Proceedings of the sixteenth annual international conference on Mobile computing and networking. ACM, 2010, pp. 1-12.

[14] M. Jain, J. I. Choi, T. Kim, D. Bharadia, S. Seth, K. Srinivasan, P. Levis, S. Katti, and P. Sinha, "Practical, real-time, full duplex wireless," in Proceedings of the 17th Annual International Conference on Mobile Computing and Networking. ACM, 2011, pp. 301-312.

[15] M. Heino, D. Korpi, T. Huusari, E. Antonio-Rodriguez, S. Venkatasubramanian, T. Riihonen, L. Anttila, C. Icheln, K. Haneda, R. Wichman, and M. Valkama, "Recent advances in antenna design and interference cancellation algorithms for in-band full duplex relays," IEEE Commun. Mag., vol. 53, no. 5, pp. 91-101, May 2015.

[16] G. Zheng, "Joint beamforming optimization and power control for fullduplex mimo two-way relay channel," IEEE Trans. Signal Process., vol. 63, no. 3, pp. 555-566, Feb 2015.

[17] N. Tran, L. Rodriguez, and T. Le-Ngoc, "Optimal power control and error performance for full-duplex dual-hop AF relaying under residual self-interference," IEEE Commun. Lett., vol. 19, no. 2, pp. 291-294, Feb 2015

[18] Z. Zhang, Z. Ma, M. Xiao, G. K. Karagiannidis, Z. Ding, and P. Fan, "Two-timeslot two-way full-duplex relaying for $5 \mathrm{~g}$ wireless communication networks," IEEE Trans. Commun., vol. 64, no. 7, pp. 2873-2887, July 2016.

[19] B. A. Ogunnaike, Random phenomena: fundamentals of probability and statistics for engineers. CRC Press, 2011.

[20] A. Jeffrey and D. Zwillinger, Table of integrals, series, and products. Academic Press, 2007.

[21] M. Ahsanullah, B. G. Kibria, and M. Shakil, Normal and Student's $t$ Distributions and Their Applications. Springer, 2014.

[22] A. Hyadi, M. Benjillali, M. S. Alouini, and D. B. da Costa, "Performance analysis of underlay cognitive multihop regenerative relaying systems with multiple primary receivers," IEEE Trans. Wireless Commun., vol. 12, no. 12, pp. 6418-6429, December 2013.

[23] M. K. Simon and M.-S. Alouini, Digital communication over fading channels. John Wiley \& Sons, 2005. 